\title{
Unveiling the Burden of Interactions Among Clinical Risk Factors for 1-Year Mortality in Hospitalized Older Patients
}

OPEN ACCESS

Edited by:

Tzvi Dwolatzky,

Technion Israel Institute of

Technology, Israel

Reviewed by:

Dan Justo,

Sheba Medical Center, Israel

Francesco Cacciatore

University of Naples Federico II, Italy

*Correspondence:

Luca Soraci

drlucasoraci89@gmail.com;

I.soraci@inrca.it

Specialty section:

This article was submitted to

Geriatric Medicine,

a section of the journal

Frontiers in Medicine

Received: 05 September 2021

Accepted: 26 October 2021

Published: 25 November 2021

Citation:

Lattanzio F, Corigliano V, Soraci L,

Fumagalli A, Onder G, Volpato $S$, Cherubini A, Ruggiero C, Cozza A,

Guarasci $F$ and Corsonello A (2021)

Unveiling the Burden of Interactions

Among Clinical Risk Factors for 1-Year

Mortality in Hospitalized Older

Patients. Front. Med. 8:771115.

doi: 10.3389/fmed.2021.771115

\begin{abstract}
Fabrizia Lattanzio ${ }^{1}$, Valentina Corigliano ${ }^{2}$, Luca Soraci ${ }^{3 *}$, Alessia Fumagalli ${ }^{4}$, Graziano Onder ${ }^{5}$, Stefano Volpato ${ }^{6,7}$, Antonio Cherubini $^{8}$, Carmelinda Ruggiero ${ }^{9}$, Annalisa Cozza ${ }^{10}$, Francesco Guarasci ${ }^{10}$ and Andrea Corsonello ${ }^{3,10}$

${ }^{1}$ Scientific Direction, Istituto Di Ricovero e Cura a Carattere Scientifico Italian National Research Centers on Aging, Ancona, Italy, ${ }^{2}$ Department of Clinical and Experimental Medicine, University of Messina, Messina, Italy, ${ }^{3}$ Unit of Geriatric Medicine, Istituto Di Ricovero e Cura a Carattere Scientifico Italian National Research Centers on Aging, Cosenza, Italy, ${ }^{4}$ Respiratory Unit, Istituto Di Ricovero e Cura a Carattere Scientifico Italian National Research Centers on Aging, Casatenovo, Italy, ${ }^{5}$ Department of Cardiovascular, Endocrine-Metabolic Diseases and Aging, IstitutoSuperiore di Sanitá, Rome, Italy, ${ }^{6}$ Department of Medical Sciences, University of Ferrara, Ferrara, Italy, ${ }^{7}$ Center for Clinical Epidemiology, School of Medicine, University of Ferrara, Ferrara, Italy, ${ }^{8}$ Geriatria, Accettazionegeriatrica e Centro di Ricerca per I'Invecchiamento, Istituto Di Ricovero e Cura a Carattere Scientifico Italian National Research Centers on Aging, Ancona, Italy, ${ }^{9}$ Orthogeriatric and Geriatric Units, Gerontology and Geriatric Sections, Department of Medicine and Surgery, University of Perugia, Perugia, Italy, ${ }^{10}$ Unit of Geriatric Pharmacoepidemiology and Biostatistics, Istituto Di Ricovero e Cura a Carattere Scientifico Italian National Research Centers on Aging, Ancona, Italy
\end{abstract}

Background: Hospitalized older patients are particularly exposed to adverse health outcomes.

Objective: In this study, we aimed at investigating the prognostic interactions between disability in basic activities of daily living (BADL), cognitive impairment, low handgrip strength, anticholinergic cognitive burden (ACB), and depression on 1-year mortality.

Setting and Subjects: Our series consisted of 503 older patients discharged from acute care hospitals.

Methods: Disability in at least one BADL, ACB, depression, cognitive impairment, and low handgrip strength was considered in the analysis. One-year mortality was investigated by Cox regression analysis and prognostic interactions among study variables were assessed by survival tree analysis.

Results: Basic activities of daily living disability, ACB, cognitive impairment, and low handgrip strength were significantly associated with 1-year mortality. Survival tree analysis showed that patients with BADL disability and high ACB carried the highest risk of poor survival [hazard ratio (HR): 16.48 (2.63-74.72)], followed by patients with BADL disability and low ACB (HR: 8.43, 95\% Cl: 1.85-38.87). Patients with cognitive impairment and no BADL disability were characterized by a lower but still significant risk of mortality (HR: 6.61, 95\% Cl: 1.51-28.97) and those with high ACB scores and good cognitive and functional performance (HR: 5.28, 95\% Cl: 1.13-24.55).

Conclusion: Basic activities of daily living dependency, cognitive impairment, and ACB score were the three main predictors of 1-year mortality among patients discharged from acute care hospitals; the interaction between BADL dependency and ACB score was 
found to significantly affect survival. Early identification of such high-risk patients may help tailor targeted interventions to counteract their detrimental effects on prognosis.

Keywords: hospitalized older patients, anticholinergic burden, functional impairment, cognitive impairment, handgrip, depression

\section{INTRODUCTION}

Hospitalized older individuals represent a complex and extremely heterogeneous portion of the geriatric population, exposed to a constant burden associated with multimorbidity, polypharmacy, and acute diseases, which may affect the overall quality of life and prognosis $(1,2)$.

Several prospective studies have identified multiple predictors of increased risk of death in this population $(3,4)$. Dependency in performing basic activities of daily living (BADL) is recognized as a contributor to mortality and recurrent hospitalization among older patients with chronic diseases $(5,6)$. Cognitive impairment is a well-known predictor of poor outcomes in geriatric populations, in terms of mortality (7). Depressive symptoms also are highly prevalent among older hospitalized patients (8) and may exert relevant negative functional and prognostic implications (9). Low handgrip strength is a dynamic indicator of poor muscle function (10) and the main risk factor for diagnosing sarcopenia (11); it was shown to predict mortality, disability, and physical functioning (12). Finally, medication burden was also associated with increased mortality (13), and exposure to anticholinergic medications is an important predictor of poor outcomes among hospitalized older patients (14-17).

Interestingly, the above risk factors are known to affect each other. For example, BADL dependency may increase the risk of depression (18). Additionally, low handgrip strength may favor the development of further physical and cognitive disability $(19,20)$. Anticholinergic cognitive burden (ACB) was also found to predict both functional and cognitive impairment among older patients discharged from acute care hospitals (14). Moreover, ACB impact on mortality was greater among patients with low handgrip strength, depressive symptoms, and functional dependency. Finally, depression was found to predict functional impairment among both community-dwelling and hospitalized older patients $(21,22)$.

Despite this bulk of evidence, BADL dependency, ACB, depression, low handgrip or cognitive impairment have only been considered as individual potentially interacting variables in the former studies $(14,15,17,23,24)$. For this reason, we aimed at providing a comprehensive investigation of the prognostic interactions involving all these risk factors simultaneously present in a population of older patients discharged from acute care hospitals, to identify the most clinically relevant combinations and improve prognostic stratification.

\section{MATERIALS AND METHODS}

This study used data from a multicenter prospective observational study carried out in seven geriatric and internal medicine wards of Italian acute care hospitals. All patients consecutively admitted to participating wards between June 2010 and May 2011 were asked to participate in the study. Exclusion criteria included age $<65$ years and unwillingness to participate in the study. After obtaining written informed consent, all participants were assessed within the first $24 \mathrm{~h}$ from hospital admission and followed up until discharge. Study researchers collected information about demographic and clinical characteristics, cognitive and functional status, and medication intake before, during hospitalization, and at discharge. Medications were coded according to the Anatomical Therapeutic and Chemical classification (25). After discharge, patients were reassessed at 3, 6, and 12 months. All ethics committees at participating institutions approved the study.

Overall, 1,120 patients were enrolled in the study. Patients with incomplete baseline data $(N=3)$ and those who died during hospitalization $(N=39)$ were excluded from our analysis. Patients with missing data for cognitive impairment $(N=218)$, depression $(N=129)$, and handgrip $(N=100)$, and those with incomplete follow-up data $(N=131)$ were also excluded, leaving a final sample of 503 patients to be included in the analysis.

Patients excluded from the study were older $(83.1 \pm 7.3$ vs. $79.4 \pm 7.0 ; p<0.001)$ and had higher prevalence of BADL disability ( 52.7 vs. $21.1 \%, p<0.001$ ), cognitive impairment ( 69.4 vs. $41.3 \%, p<0.001$ ), depression ( 46.1 vs. $35.0 \%, p=0.005$ ), and higher average ACB score $(1.4 \pm 1.4$ vs. $1.2 \pm 1.1, p<0.001)$ compared to those included in the study.

\section{Exposure Variables}

Comprehensive geriatric assessment and medication data were collected at the time of discharge. Cognitive impairment was defined as having age- and education-adjusted Mini-Mental State Examination < 24 (26); the number of BADL (27) was calculated at discharge, and an analytic variable was created to identify patients with dependency in at least 1 BADL. Depression was defined as having a 15-item Geriatric Depression Scale (28) score $>5$. Exposure to anticholinergic medications was quantified by calculating the anticholinergic cognitive burden (ACB) score (29), which was chosen because it was externally validated (30) and considered more accurate in the evaluation of central anticholinergic burden compared with other tools (31). The exposure variable based on the calculation of $\mathrm{ACB}$ score at discharge was categorized as follows: low-medium anticholinergic burden $(\mathrm{ACB}=0$ or 1 and $\mathrm{ACB}=0$ for patients taking no anticholinergic medications) and high anticholinergic burden (ACB score 2 or more). Muscle strength was assessed by handgrip strength, measured by calibrated hand dynamometer (North Coast Hydraulic Hand Dynamometer, North Coast Medical Inc, Morgan Hill, CA, USA), as previously described (32). According to the revised European Working Group on Sarcopenia in Older People 2 criteria, low muscle strength was 
classified as handgrip $<27 \mathrm{~kg}$ in men and $<16 \mathrm{~kg}$ in women (11). Finally, the cumulative number of exposure variables (low handgrip, BADL disability, depression, cognitive impairment, and high ACB) was also calculated and included in the analysis.

\section{Outcome}

The outcome of this study was 1-year mortality. Data on living status during follow-up were obtained by interviewing the patients and/or their formal and/or informal caregivers. The time from the day of the study enrollment through the day of death was used as the time to failure variable for the model. Survivors were censored at the end of the follow-up. About patients who died during the follow-up period, date and place of death were retrieved by relatives or caregivers. The municipal registers were consulted when neither patients or relatives nor caregivers could be contacted.

\section{Covariates}

Age, sex, number of diseases at discharge, and number of medications prescribed at discharge were considered as potential confounders in the analysis. Selected diagnoses known to affect prognosis in older patients (hypertension, heart failure, coronary artery disease, atrial fibrillation, peripheral artery disease, diabetes mellitus, chronic obstructive pulmonary disease, chronic kidney disease, stroke, dementia, and cancer) were also included in the preliminary analysis. Finally, to account for the continuity of exposure to anticholinergic drugs during the first follow-up period after discharge, the $\mathrm{ACB}$ score at 3-month follow-up was also considered as a potential confounder in the analysis.

\section{Statistical Analysis}

First, we compared survivors and patients who died during follow-up concerning study variables and covariates. Continuous variables were reported as mean $\pm \mathrm{SD}$. Categorical variables were expressed as several cases (percentage). To compare the characteristics of the patients, according to 1-year survival status, we used the Student's $t$-test for continuous variables and the chi-squared test for categorical ones.

The relative risk of 1-year mortality related to either single study risk factors (low handgrip, cognitive impairment, functional impairment, depression, and ACB score) or the cumulative number of risk factors was then investigated by Cox regression analysis. We fitted three different Cox regression models: model A, adjusted for age and sex; model B, adjusted for age, sex, number of medications, and number of diagnoses; and model $\mathrm{C}$, adjusted for age, sex, number of medications, and individual diagnoses associated with mortality in the preliminary analysis (heart failure, hypertension, coronary artery disease, cancer, and atrial fibrillation) instead of several medications. Models $\mathrm{B}$ and $\mathrm{C}$ were also repeated after adjusting for ACB score at 3-month follow-up.

Risk factors significantly associated with mortality in adjusted models were further analyzed by Venn diagram to investigate their overlapping. Therefore, to improve the graphical representation of the predictive models, we fitted a survival tree model based on study risk factors significantly associated with mortality in Cox regression models. Survival trees represent peculiar non-parametric alternatives to (semi)parametric models; they are characterized by extreme flexibility that allows automatic detection of certain types of interactions without specifying them beforehand. In this study, the splitting criterion was based on a node deviance measure between a saturated model log-likelihood and a maximized log-likelihood as proposed by Leblanc and Crowley (33), with further node adjustment to simplify the graphical representation of the fitted survival tree. To assess the performance of the fitted survival tree, the leaf node membership was added as a categorical variable in a Cox regression model using the node with the best survival as the reference category. Bootstrap model validation (1,000 resamplings) was performed to limit the bias of the estimates. The accuracy performance of the model was assessed by calculating the C-index value and 95\%CI for leaf node membership and compared with that of individual study risk factors or the cumulative number of risk factors.

We also planned to perform sensitivity analyses. To account for potential residual confounding, survival tree analysis was repeated including patients with available data for risk factors significantly associated with the outcome. C-index of leaf node membership was separately calculated for each survival tree. Finally, survival tree analysis was repeated by forcing into survival tree analysis risk factor(s) not significantly associated with mortality.

Analyses were performed using SPSS (version 26.0, SPSS, Chicago, IL, USA) and rpart (34) and partykit (35) packages of R (version 4.0.2, R Foundation for Statistical Computing, Vienna, Austria).

\section{RESULTS}

Baseline characteristics according to 1-year mortality are depicted in Table 1. Among the 503 study participants (mean age: $79.4 \pm 7.0,50.7 \%$ women), 69 patients (13.7\%) died during the follow-up. Patients who died were older, malnourished, and had a higher number of medications and diagnoses, a greater prevalence of heart failure, coronary artery disease, cancer, and atrial fibrillation, and a lower prevalence of hypertension. Patients who died during the follow-up were also characterized by a higher prevalence of low handgrip, cognitive impairment, BADL disability, depression, and high anticholinergic burden. ACB medications prescribed at discharge according to 1-year mortality are reported in Supplementary Table 1.

Cox regression analysis showed that all study risk factors but depression were associated with increased 1-year mortality in age- and sex-adjusted models, and similar findings were obtained in models $\mathrm{B}$ and $\mathrm{C}$ after adjusting for potential confounders (Table 2). Such results were confirmed after including ACB score at 3-month follow-up in fully adjusted models $\mathrm{B}$ and $\mathrm{C}$; the corresponding figures (hazard ratio and 95\% CI) for model C including $\mathrm{ACB}$ score at 3 months were 4.88 (2.60-9.16) for ACB score, 2.42 (1.48-3.98) for BADL disability, 1.87 (1.12-3.12) for cognitive impairment, 1.78 (1.03-3.01) for low handgrip strength, and $1.31(0.76-2.26)$ for depression. 
TABLE 1 | Demographic and clinical characteristics of discharged patients grouped by 1-year mortality.

\begin{tabular}{|c|c|c|c|c|}
\hline & $\begin{array}{l}\text { All patients } \\
(n=503)\end{array}$ & $\begin{array}{l}\text { Survivors } \\
(n=434)\end{array}$ & $\begin{array}{c}\text { Died } \\
(n=69)\end{array}$ & $P$ \\
\hline Age, mean ( \pm SD) & $79.4 \pm 7.0$ & $78.9 \pm 7.0$ & $82.8 \pm 6.3$ & $<0.001$ \\
\hline Heart failure, $n(\%)$ & $142(28.2 \%)$ & 109 (25.1\%) & $33(47.8 \%)$ & $<0.001$ \\
\hline Hypertension, $n$ (\%) & 399 (79.3\%) & 356 (82.0\%) & 43 (62.3\%) & $<0.001$ \\
\hline COPD, $n(\%)$ & $206(40.9 \%)$ & 171 (39.4\%) & 35 (50.7\%) & 0.10 \\
\hline CKD, $n(\%)$ & $243(50.2 \%)$ & $204(48.6 \%)$ & $39(60.9 \%)$ & 0.09 \\
\hline Cancer, $n(\%)$ & $70(13.9 \%)$ & $43(9.9 \%)$ & 27 (39.1\%) & $<0.001$ \\
\hline Diabetes, $n(\%)$ & $154(30.6 \%)$ & $132(30.4 \%)$ & 22 (31.9\%) & 0.92 \\
\hline Dementia, $n(\%)$ & $53(10.5 \%)$ & $47(10.8 \%)$ & $6(8.6 \%)$ & 0.745 \\
\hline \multicolumn{5}{|l|}{ Study risk factors } \\
\hline ACB score & & & & $<0.001$ \\
\hline $0-1$ & 355 (70.6\%) & $319(73.5 \%)$ & 36 (52.2\%) & \\
\hline 2 or more & $148(29.4 \%)$ & $115(26.5 \%)$ & $33(47.8 \%)$ & \\
\hline BADL disability & $106(21.1 \%)$ & $76(17.5 \%)$ & 30 (43.5\%) & $<0.001$ \\
\hline Cognitive impairment & $208(41.3 \%)$ & $167(38.5 \%)$ & 41 (59.4\%) & 0.002 \\
\hline Low handgrip strength & $233(46.3 \%)$ & $191(44.0 \%)$ & 42 (60.9\%) & 0.01 \\
\hline Depression & 176 (35.0\%) & 147 (33.9\%) & 29 (42.0\%) & 0.24 \\
\hline
\end{tabular}

$p$-value from $t$-test or $x^{2}$ test as appropriate. Data are expressed as mean $\pm S D$ or $n$ and percentage as appropriate.

$B A D L$, basic activities of daily living; CAD, coronary artery disease; PAD, peripheral artery disease; COPD, chronic obstructive pulmonary disease; CKD, chronic kidney disease.

TABLE 2 | The Cox regression analysis of study risk factors in relation to 1 -year mortality.

\begin{tabular}{lccr}
\hline & $\begin{array}{c}\text { Model A } \\
\text { HR (95\% } \mathbf{C l})\end{array}$ & $\begin{array}{c}\text { Model B } \\
\text { HR (95\% Cl) }\end{array}$ & $\begin{array}{c}\text { Model C } \\
\text { HR (95\%Cl) }\end{array}$ \\
\hline ACB score 0-1 & - & - & - \\
ACB score 2 or more & $2.17(1.33-3.43)$ & $1.66(1.01-2.80)$ & $1.61(1.00-2.71)$ \\
Low handgrip & $1.85(1.03-3.33)$ & $1.77(1.02-3.18)$ & $1.72(1.01-3.20)$ \\
Cognitive impairment & $1.94(1.19-3.17)$ & $1.90(1.16-3.11)$ & $2.01(1.21-3.33)$ \\
BADL disability & $2.95(1.79-4.86)$ & $2.75(1.65-4.56)$ & $2.50(1.53-4.08)$ \\
Depression & $1.33(0.80-2.19)$ & $1.26(0.76-2.08)$ & $1.31(0.76-2.36)$ \\
\hline
\end{tabular}

Model A: adjusted for age and gender; Model B: adjusted for age, sex, number of medications and number of diagnoses; and Model C: as for model B with heart failure, hypertension, coronary artery disease, cancer, and atrial fibrillation instead of number of diagnoses.

$A C B$, anticholinergic cognitive burden; $B A D L$, basic activities of daily living; $H R$, hazard ratio.

Overlapping among study risk factors significantly associated with mortality is shown in Figure 1. Low handgrip strength was the most common risk factor in the study population (55.6\%), followed by cognitive impairment (41.3\%), high ACB (29.4\%), and BADL disability (21.1\%). In contrast to high $\mathrm{ACB}$, low handgrip, and cognitive impairment, the presence of BADL disability was rarely observed alone and was often observed in combination with other risk factors. The most common combinations of study risk factors involved cognitive impairment and low handgrip strength (13.7\%), BADL disability, cognitive impairment, and low handgrip strength (7.7\%), low handgrip and high ACB (5.4\%), and cognitive impairment, low handgrip, and high ACB (4.6\%).

Results obtained by survival tree analysis are reported in Figure 2. Node 5 (patients with no BADL disability, no cognitive impairment, $\mathrm{ACB}$ score $<2$, and normal handgrip, $n=113$ ) showed the lowest mortality and was considered as the reference 


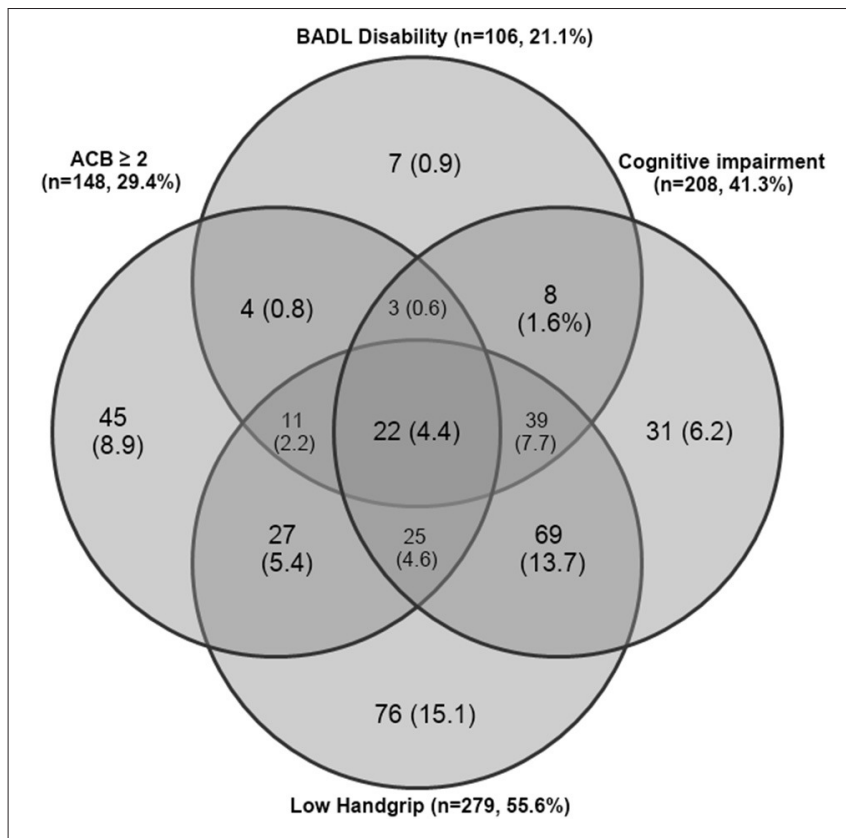

FIGURE 1 | Venn diagram showing overlapping of study risk factors significantly associated with mortality in the study population.

category in the Cox regression models. The highest risk of mortality was observed among patients belonging to Node 11 (BADL disability and ACB score of 2 or more) and Node 10 (BADL disability and ACB score < 2). Node 5 (no BADL disability, no cognitive impairment, $\mathrm{ACB}$ score $<2$, and normal handgrip), and Node 6 (no BADL disability, no cognitive impairment, $\mathrm{ACB}$ score $<2$, and impaired handgrip) shared the lowest mortality risk, while Nodes 7 and 8 showed intermediate mortality risk profiles. Descriptive features of leaf node groups are reported in Supplementary Table 2.

The analysis including the number of risk factors or leaf node membership instead of single study risk factors showed that the coexistence of 2 or more study risk factors was significantly associated with mortality (Figure 3). Leaf node membership showed a progressive increase in mortality risk of death starting from Node 6.

The leaf node membership showed a good predictive accuracy (C-index: 0.812; 95\% CI: 0.761-0.852), which was significantly better compared to that observed with several risk factors and individual risk factors ( $p<0.05$, see Table 3). Similar results were obtained by sensitivity analyses to fit a family of survival trees among patients with available BADL $(n=807)$, cognitive impairment $(n=670)$, handgrip $(n=621)$, and ACB score $(n=807)$ data (Supplementary Figure 1). The Cindex of single survival trees was substantially comparable to that of the main survival tree (mean C-index 0.81, $p=0.85$ ). Forcing the inclusion of depression in the survival tree analysis allowed us to identify a subgroup of patients with BADL disability and depression interacting in predicting 1-year mortality (Supplementary Figure 2).

\section{DISCUSSION}

In this prospective cohort study, we used a tree-based risk algorithm to identify the most relevant prognostic interactions among selected risk factors in older patients discharged from acute care hospitals. The survival tree analysis allowed the identification of selected combinations of risk factors leading to different prognostic outcomes concerning 1-year mortality. Patients with BADL disability and ACB score $\geq$ 2 (Node 11) were characterized by the highest mortality, followed by individuals with BADL disability and $\mathrm{ACB}$ score $<2$ (node 10). Node 8 (cognitive impairment with no BADL disability) and Node 7 (ACB score of 2 or more with no cognitive impairment and no BADL disability) were characterized by a lower but still significantly increased mortality risk. Finally, cognitive impairment was highly prevalent among high-risk nodes but did not show any other significant interaction with either functional impairment or anticholinergic burden.

Functional impairment was an independent predictor of mortality in this population, thus confirming results from previous studies $(36,37)$; functional disability may be the expression of both presence and severity of multiple diseases on the health of individual and then characterize individuals at higher risk of poor long-term survival (38). Furthermore, dependency in at least one BADL showed a significant interaction with high ACB.

Patients with BADL disability and ACB of two or more were characterized by a 16 -fold increased risk of mortality compared to the reference category, which confirms previous evidence regarding the need of moderating the use of anticholinergic medications in patients with functional impairment (17), even in the setting of this study considering a wider set of potential risk factors compared to the former one (17). The anticholinergic burden may, in fact, contribute to the effects of functional impairment on survival in older patients, by increasing the vulnerability of functionally impaired individuals to adverse drug reactions (39). Moreover, the anticholinergic burden has been shown to impair gait and balance and contribute to the risk of recurrent falls (40), representing a major cause of functional disability and death $(14,16)$. On the other side, anticholinergic medications may impair physical performance and worsen BADL dependency in patients with pre-existing disabilities, which might exponentially increase the risk of death (41).

Interestingly, depression seems to play a minor prognostic role in this study. Its prognostic weight was detected only in a small proportion of individuals after performing sensitivity analyses. Nevertheless, when forcing depression in the survival tree we could intercept a subgroup of patients with BADL disability and low ACB in which depression played an adjunctive negative prognostic role. At variance, the lack of interaction between BADL disability and either cognitive impairment or handgrip strength suggests that all these risk factors may impact prognosis through independent pathways.

Among patients with BADL independence, cognitive impairment was significantly associated with the outcome (Node 8), thus confirming previous evidence regarding this 


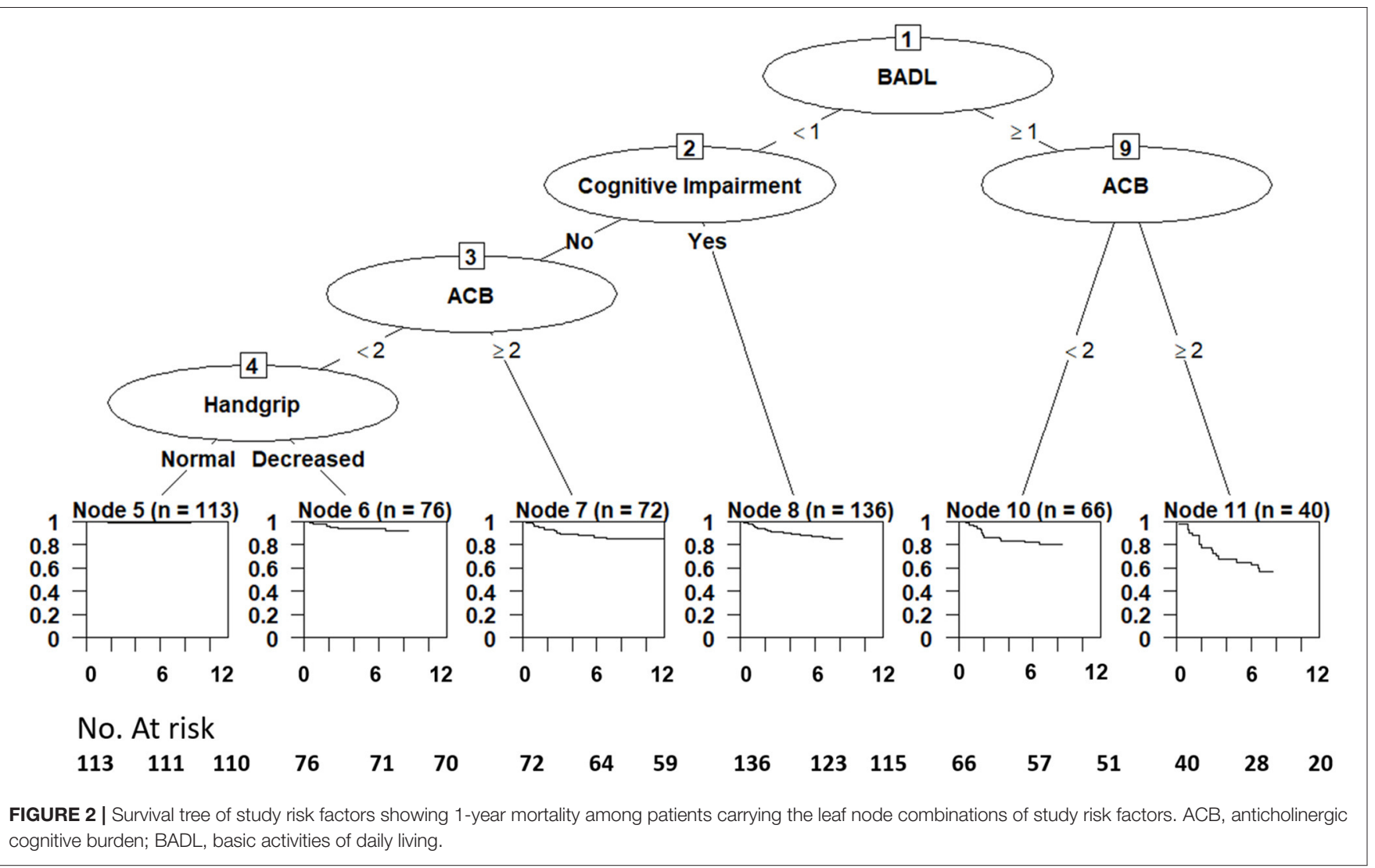

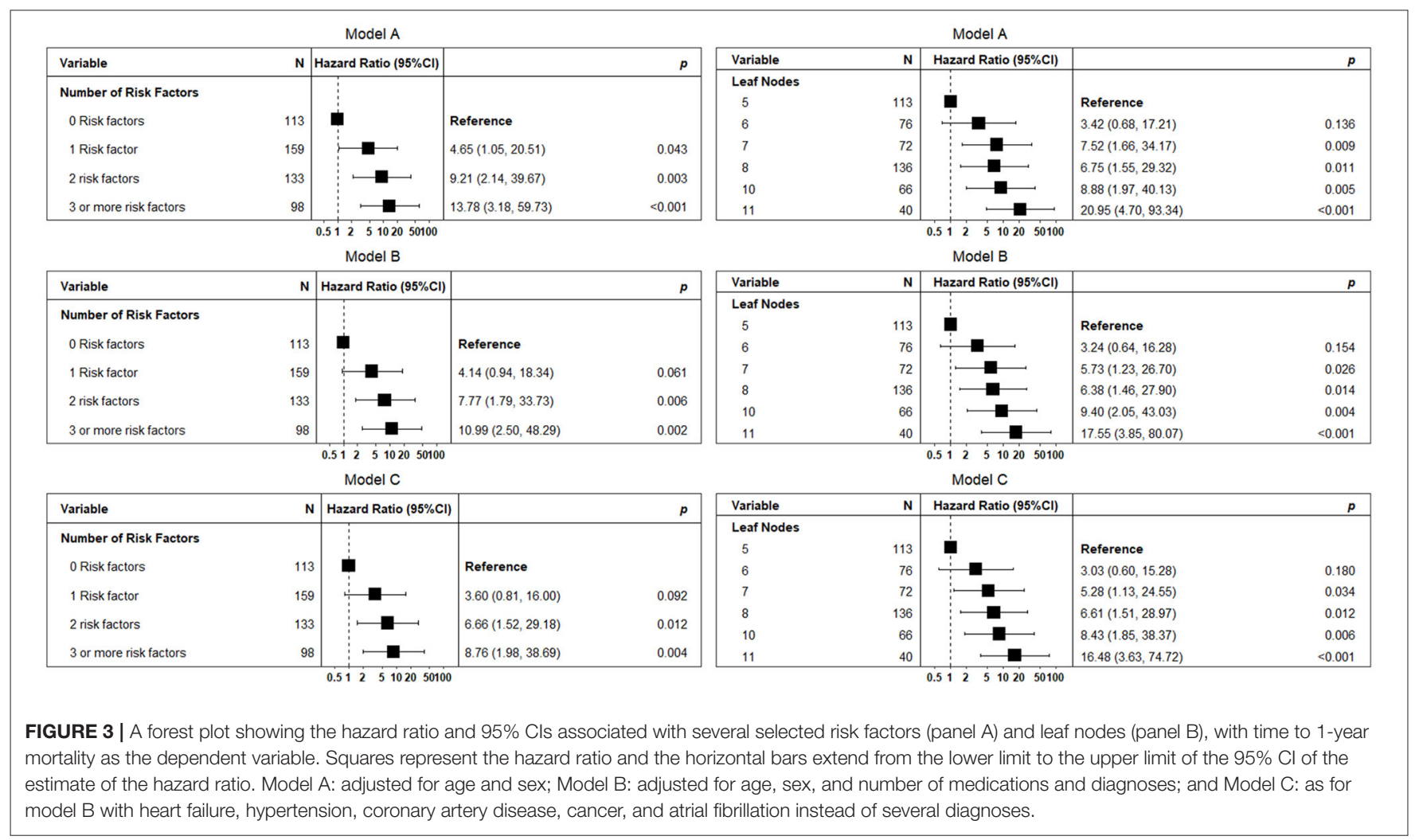


TABLE 3 | C-index in predicting 1-year mortality obtained by leaf nodes membership from survival tree analysis compared to individual risk factors or number of risk factors.

\begin{tabular}{|c|c|c|}
\hline & Age and sex-adjusted C-index & $P$ value \\
\hline Depression & $0.661(0.611-0.720)$ & $<0.001$ \\
\hline Low handgrip & $0.685(0.626-0.743)$ & $<0.001$ \\
\hline Cognitive impairment & $0.701(0.644-0.754)$ & $<0.001$ \\
\hline ACB score $\geq 2$ & $0.699(0.642-0.755)$ & $<0.001$ \\
\hline BADL disability & $0.718(0.661-0.774)$ & 0.007 \\
\hline Number of risk factors & $0.736(0.685-0.787)$ & 0.018 \\
\hline Leaf node membership & $0.812(0.761-0.852)$ & - \\
\hline
\end{tabular}

$A C B$, anticholinergic cognitive burden; $B A D L$, basic activities of daily living.

association, even in patients without diagnosis of dementia $(42,43)$; possible explanations for this association include poor adherence to medications, increased drug burden due to the treatment of cognitive disturbances, and increased incidence of frailty (43). Anticholinergic medications carried a less striking, but still important prognostic weight in patients with BADL independence; drugs with anticholinergic properties may increase the risk of cognitive impairment and dementia, falls and mortality $(15,17,24)$. Furthermore, these drugs may have cardiovascular and neurological effects $(44,45)$, such as arrhythmias, syncope, hallucinations, and seizures, that might provoke serious adverse events, ultimately leading to death (46).

In the end, low handgrip strength, despite its high prevalence, did not independently contribute to the risk of mortality in the survival tree analysis, thus suggesting that its detrimental consequences are probably mediated by other factors.

The findings of this study may have several clinical and prognostic implications. The major prognostic interaction involved functional disability and $\mathrm{ACB}$ score, consequently, the assessment of functional status and revision of drug treatment during hospital stay should be important cornerstones of clinical evaluation of older hospitalized patients, to identify highrisk patients to whom reserve individualized pharmacological treatments. In this regard, the ACB score may represent a useful index of both pharmacological and mortality risk and may help predict future functional decline (14). A high ACB score was a significant predictor of mortality among patients with preserved functional and cognitive abilities; for this reason, careful and tailored prescribing and regular monitoring of anticholinergic medications should not be scotomized when dealing with these patients.

\section{Limitations}

This study has some limitations. First, given the observational design, confounding by indication is a relevant limitation in the study. Even if results were confirmed in sensitivity analyses, a greater prevalence of depression, cognitive, and functional impairment, and a greater ACB score were found among patients excluded because of missing data. Additionally, missing may have contributed to the observation of a less relevant role of depression and handgrip in survival tree analyses. Second, the results of the survival tree analysis may lack the precision of estimates due to the small sample size, and wide CIs are in keeping with this hypothesis. Third, our results identify variables that by themselves may influence the outcome, and we could not account for illness severity, duration, and management of individual diagnoses. Fourth, the short duration of follow-up does not allow to optimally explore the long-term association between study risk factors and mortality. Additionally, our database included only limited information about the duration of exposure to ACB drugs and we could only calculate the ACB score at a 3-month followup. While adjusting the analysis for the ACB score at 3 months did not affect our main findings, we could not fully explore the effect of long-lasting exposure to anticholinergic medications compared with a shorter duration of treatment. Finally, the lack of information about post-acute care utilization did not allow us to investigate the impact of post-discharge care on study results. Finally, our results apply to the population of older patients discharged from acute care hospitals and cannot be generalized to the general older population.

On the other hand, the main strengths of our study are represented by the inclusion of a real-world unselected population of hospitalized older patients, the thorough evaluation of medications, and the use of a comprehensive geriatric assessment to explore the independent effect of several risk factors. In addition, this is the first study that identified several important interactions between previously investigated risk factors taken altogether by using the survival tree approach. Furthermore, the high stability and reproducibility of survival trees in sensitivity analyses and the high level of predictive accuracy estimates add further significance to study findings. If confirmed on larger studies, such interactions may have significant clinical and prognostic implications, which might suggest the need of using appropriate interventions to counteract the negative effects of selected risk factors on outcomes of patients. In this regard, hospital physicians should consider the assessment of $\mathrm{ACB}$ and functional and cognitive status as very important components of clinical practice.

\section{Conclusion and Implications}

Functional impairment was the main predictor of 1-year mortality among hospitalized older patients and was found to interact with ACB score. Among patients with preserved functional capacity, cognitive impairment and anticholinergic 
burden were the main predictors of 1-year mortality. These findings support the importance of a careful evaluation of functional and cognitive status, and anticholinergic burden among older patients discharged from acute care hospitals to de-prescribe anticholinergic medications whenever possible, especially in patients carrying a selected combination of risk factors.

\section{DATA AVAILABILITY STATEMENT}

The datasets presented in this study can be found in online repositories. The names of the repository/repositories and accession number(s) can be found below: Data are available for CRIME study researchers at IRCCS INRCA (www.inrca.it).

\section{ETHICS STATEMENT}

The studies involving human participants were reviewed and approved by Ethics Committee of the Catholic University of Rome (Project identification code: P/582/CE/2009). The patients/participants provided their written informed consent to participate in this study.

\section{AUTHOR CONTRIBUTIONS}

ACor, VC, LS, FG, ACoz, and AF participated in data analysis, manuscript writing, and revising and manuscript approval. GO, SV, CR, AChe, and ACor participated in data collection and writing, revising, and approving manuscript. FL participated in writing the manuscript, revising it for important intellectual

\section{REFERENCES}

1. Sganga F, Landi F, Volpato S, Cherubini A, Ruggiero C, Corsonello A, et al. Predictors of rehospitalization among older adults: results of the CRIME Study. GeriatrGerontol Int. (2017) 17:1588-92. doi: 10.1111/ggi. 12938

2. Sganga F, Landi F, Ruggiero C, Corsonello A, Vetrano DL, Lattanzio F, et al. Polypharmacy and health outcomes among older adults discharged from hospital: results from the CRIME study. GeriatrGerontol Int. (2015) 15:141-6. doi: 10.1111/ggi.12241

3. Levine SK, Sachs GA, Jin L, Meltzer D. A prognostic model for 1-year mortality in older adults after hospital discharge. Am J Med. (2007) 120:45560. doi: 10.1016/j.amjmed.2006.09.021

4. Fompeyrine C, Abderhalden LA, Mantegazza N, Hofstetter N, Bieri-Brüning G, Bischoff-Ferrari HA, et al. Frailty is associated with increased mortality in older adults 12 months after discharge from post-acute care in swiss nursing homes. J Frailt Aging. (2020) 10: 233-6. doi: 10.1038/s41418-018-0232-2

5. Bossola M, Di Stasio E, Antocicco M, Pepe G, Tazza L, Zuccalà G, et al. Functional impairment is associated with an increased risk of mortality in patients on chronic hemodialysis. BMC Nephrol. (2016) 17:72. doi: 10.1186/s12882-016-0302-y

6. Dunlay SM, Manemann SM, Chamberlain AM, Cheville AL, Jiang R, Weston SA, et al. Activities of daily living and outcomes in heart failure. Circul Heart Fail. (2015) 8:261-7. doi: 10.1161/CIRCHEARTFAILURE.114. 001542

7. Fogg C, Meredith P, Bridges J, Gould GP, Griffiths P. The relationship between cognitive impairment, mortality and discharge characteristics in a large cohort of older adults with unscheduled admissions to an acute content, and approval. All authors contributed to the article and approved the submitted version.

\section{FUNDING}

This study was funded by Italian National Research Center on Aging (IRCCS INRCA) intramural research funds. The CRiteria to assess Inappropriate Medication use among Elderly complex patients (CRIME) project was partially supported by a grant from the Italian Ministry of Health (GR-2007 685638).

\section{ACKNOWLEDGMENTS}

The complete list of CRIME study investigators was previously published elsewhere (47). The CRIME study group: Gemelli Hospital, Centro Medicinadell'Invecchiamento, UniversitàCattolica del SacroCuore, Rome, Italy; University of Perugia; University of Ferrara; Italian National Research Center on Aging (IRCCS INRCA) Ancona, Cosenza, Fermo, and Rome. The authors are grateful to Romano Firmani and Moreno Nacciariti for their skillful technical support.

\section{SUPPLEMENTARY MATERIAL}

The Supplementary Material for this article can be found online at: https://www.frontiersin.org/articles/10.3389/fmed. 2021.771115/full\#supplementary-material hospital: a retrospective observational study. Age Ageing. (2017) 46:794801. doi: 10.1093/ageing/afx022

8. Prina AM, Cosco TD, Dening T, Beekman A, Brayne C, Huisman M. The association between depressive symptoms in the community, non-psychiatric hospital admission and hospital outcomes: a systematic review. J Psychosom Res. (2015) 78:25-33. doi: 10.1016/j.jpsychores.2014.11.002

9. Pierluissi E, Mehta KM, Kirby KA, Boscardin WJ, Fortinsky RH, Palmer $\mathrm{RM}$, et al. Depressive symptoms after hospitalization in older adults: function and mortality outcomes. J Am Geriatr Soc. (2012) 60:225462. doi: $10.1111 /$ jgs. 12008

10. Norman K, Stobäus N, Gonzalez MC, Schulzke JD, Pirlich M. Hand grip strength: outcome predictor and marker of nutritional status. Clinic Nutri. (2011) 30:135-42. doi: 10.1016/j.clnu.2010.09.010

11. Cruz-Jentoft AJ, Bahat G, Bauer J, Boirie Y, Bruyère O, Cederholm T, et al. Sarcopenia: revised European consensus on definition and diagnosis. Age Ageing. (2019) 48:16-31. doi: 10.1093/ageing/afy169

12. Bohannon RW. Muscle strength: clinical and prognostic value of handgrip dynamometry. Curr Opin Clin Nutr Metab Care. (2015) 18:46570. doi: $10.1097 / \mathrm{MCO} .0000000000000202$

13. Carr E, Federman A, Dzahini O, Dobson RJ, Bendayan R. A multidimensional measure of polypharmacy for older adults using the health and retirement study. Sci Rep. (2021) 11:8783. doi: 10.1038/s41598-021-86331-x

14. Brombo G, Bianchi L, Maietti E, Malacarne F, Corsonello A, Cherubini A, et al. Association of anticholinergic drug burden with cognitive and functional decline over time in older inpatients: results from the CRIME project. Drugs Aging. (2018) 35:917-24. doi: 10.1007/s40266-018-0584-9

15. Corsonello A, Cozza A, D’Alia S, Onder G, Volpato S, Ruggiero C, et al. The excess mortality risk associated with anticholinergic burden among older 
patients discharged from acute care hospital with depressive symptoms. Eur J Intern Med. (2019) 61:69-74. doi: 10.1016/j.ejim.2018.11.004

16. Gagliardi C, Corsonello A, Di Rosa M, Fabbietti P, Cherubini A, Mercante $\mathrm{O}$, et al. Preadmission functional decline predicts functional improvement among older patients admitted to acute care hospital. J Gerontol A BiolSci Med Sci. (2018) 73:1363-9. doi: 10.1093/gerona/glx211

17. Lattanzio F, Onder G, La Fauci MM, Volpato S, Cherubini A, Fabbietti P, et al. Anticholinergic burden is associated with increased mortality in older patients with dependency discharged from hospital. J Am Med Dir Assoc. (2018) 19:942-7. doi: 10.1016/j.jamda.2018.06.012

18. Habtamu K, Medhin G, Selamu M, Tirfessa K, Hanlon C, Fekadu A. Functional impairment among people diagnosed with depression in primary healthcare in rural Ethiopia: a comparative cross-sectional study. Int J Ment Health Syst. (2019) 13:50. doi: 10.1186/s13033-019-0305-8

19. Giampaoli S, Ferrucci L, Cecchi F, Lo Noce C, Poce A, Dima F, et al. Hand-grip strength predicts incident disability in non-disabled older men. Age Ageing. (1999) 28:283-8. doi: 10.1093/ageing/28.3.283

20. Kim KH, Park SK, Lee DR, Lee J. The relationship between handgrip strength and cognitive function in elderly Koreans over 8 years: a prospective population-based study using Korean longitudinal study of ageing. Korean J Fam Med. (2019) 40:9-15. doi: 10.4082/kjfm.17.0074

21. Stuck AE, Walthert JM, Nikolaus T, Büla CJ, Hohmann C, Beck JC. Risk factors for functional status decline in community-living elderly people: a systematic literature review. Soc Sci Med. (1999) 48:44569. doi: 10.1016/S0277-9536(98)00370-0

22. Lenze EJ, Schulz R, Martire LM, Zdaniuk B, Glass T, Kop WJ, et al. The course of functional decline in older people with persistently elevated depressive symptoms: longitudinal findings from the cardiovascular health study. J Am Geriatr Soc. (2005) 53:569-75. doi: 10.1111/j.1532-5415.2005.53202.x

23. D'Alia S, Guarasci F, Bartucci L, Caloiero R, Guerrieri ML, Soraci L, et al. Hand grip strength may affect the association between anticholinergic burden and mortality among older patients discharged from hospital. Drugs Aging. (2020) 37:447-55. doi: 10.1007/s40266-020-00766-x

24. Lattanzio F, Corica F, Schepisi R, Amantea D, Bruno F, Cozza A, et al. Anticholinergic burden and 1-year mortality among older patients discharged from acute care hospital. GeriatrGerontol Int. (2018) 18:70513. doi: 10.1111 /ggi.13234

25. Pahor M, Chrischilles EA, Guralnik JM, Brown SL, Wallace RB, Carbonin P. Drug data coding and analysis in epidemiologic studies. Eur J Epidemiol. (1994) 10:405-11. doi: 10.1007/BF01719664

26. Folstein MF, Folstein SE, McHugh PR. "Mini-mental state”. J Psychiatr Res. (1975) 12:189-98. doi: 10.1016/0022-3956(75)90026-6

27. Katz S, Ford AB, Moskowitz RW, Jackson BA, Jaffe MW. Studies of illness in the aged. the index of adl: a standardized measure of biological and psychosocial function JAMA. (1963) 185:914-9. doi: 10.1001/jama.1963.03060120024016

28. Lesher EL, Berryhill JS. Validation of the geriatric depression scale-short form among inpatients. J Clinic Psychol. (1994) 50:256-60. doi: 10.1002/1097-4679(199403)50:2\&lt;256::AIDJCLP2270500218\&gt;3.0.CO;2-E

29. Boustani M, Campbell N, Munger S, Maidment I, Fox C. Impact of anticholinergics on the aging brain: a review and practical application. Aging health. (2008) 4:311-20. doi: 10.2217/1745509X.4.3.311

30. Fox C, Richardson K, Maidment ID, Savva GM, Matthews FE, Smithard D, et al. Anticholinergic medication use and cognitive impairment in the older population: the medical research council cognitive function and ageing study. J Am Geriatr Soc. (2011) 59:1477-83. doi: 10.1111/j.1532-5415.2011.03491.x

31. Salahudeen MS, Duffull SB, Nishtala PS. Anticholinergic burden quantified by anticholinergic risk scales and adverse outcomes in older people: a systematic review. BMC Geriatr. (2015) 15:31. doi: 10.1186/s12877-015-0029-9

32. Hillman TE, Nunes QM, Hornby ST, Stanga Z, Neal KR, Rowlands BJ, et al. A practical posture for hand grip dynamometry in the clinical setting. Clinic Nutri. (2005) 24:224-8. doi: 10.1016/j.clnu.2004.09.013

33. LeBlanc $M$, Crowley J. Relative risk trees for censored survival data. Biometrics. (1992) 48:411-25. doi: 10.2307/2532300

34. Therneau T, Atkinson B, Ripley B, Ripley MB. Package 'rpart';. Available online: cranmaicacuk/web/packages/rpart/rpartpdf, (accessed on 7 February 2021). 2015.
35. Hothorn T. ZeileisAJTJoMLR. partykit: A modular toolkit for recursive partytioning in $R$. (2015).

36. Millán-Calenti JC, Tubío J, Pita-Fernández S, González-Abraldes I, Lorenzo T, Fernández-Arruty T, et al. Prevalence of functional disability in activities of daily living (ADL), instrumental activities of daily living (IADL) and associated factors, as predictors of morbidity and mortality. Arch Gerontol Geriatr. (2010) 50:306-10. doi: 10.1016/j.archger.2009.04.017

37. Landi F, Liperoti R, Russo A, Capoluongo E, Barillaro C, Pahor M, et al. Disability, more than multimorbidity, was predictive of mortality among older persons aged 80 years and older. J ClinEpidemiol. (2010) 63:7529. doi: 10.1016/j.jclinepi.2009.09.007

38. Wang X-X, Lin W-Q, Chen X-J, Lin Y-Y, Huang L-L, Zhang S-C, et al. Multimorbidity associated with functional independence among communitydwelling older people: a cross-sectional study in Southern China. Health Qual Life Outcomes. (2017) 15:73. doi: 10.1186/s12955-017-0635-7

39. Cullinan S, O'Mahony D, O'Sullivan D, Byrne S. Use of a frailty index to identify potentially inappropriate prescribing and adverse drug reaction risks in older patients. Age Ageing. (2016) 45:115-20. doi: 10.1093/ageing/afv166

40. Zia A, Kamaruzzaman S, Myint PK, Tan MP. Anticholinergic burden is associated with recurrent and injurious falls in older individuals. Maturitas. (2016) 84:32-7. doi: 10.1016/j.maturitas.2015.10.009

41. Landi F, Russo A, Liperoti R, Cesari M, Barillaro C, Pahor M, et al. Anticholinergic drugs and physical function among frail elderly population. Clin Pharmacol Ther. (2007) 81:235-41. doi: 10.1038/sj.clpt.6100035

42. Sachs GA, Carter R, Holtz LR, Smith F, Stump TE, Tu W, et al. Cognitive impairment: an independent predictor of excess mortality. Ann Intern Med. (2011) 155:300-8. doi: 10.7326/0003-4819-155-5-201109060-00007

43. Perna L, Wahl H-W, Mons U, Saum K-U, Holleczek B, Brenner H. Cognitive impairment, all-cause and cause-specific mortality among non-demented older adults. Age Ageing. (2015) 44:445-51. doi: 10.1093/ageing/afu188

44. Myint PK, Fox C, Kwok CS, Luben RN, Wareham NJ, Khaw K-T. Total anticholinergic burden and risk of mortality and cardiovascular disease over 10 years in 21,636 middle-aged and older men and women of EPIC-Norfolk prospective population study. Age Ageing. (2015) 44:21925. doi: 10.1093/ageing/afu185

45. Vetrano DL, La Carpia D, Grande G, Casucci P, Bacelli T, Bernabei R, et al. Anticholinergic medication burden and 5-year risk of hospitalization and death in nursing home elderly residents with coronary artery disease. J Am Med Dir Assoc. (2016) 17:1056-9. doi: 10.1016/j.jamda.2016.07.012

46. Collamati A, Martone AM, Poscia A, Brandi V, Celi M, Marzetti E, et al. Anticholinergic drugs and negative outcomes in the older population: from biological plausibility to clinical evidence. Aging Clin Exp Res. (2016) 28:2535. doi: 10.1007/s40520-015-0359-7

47. Tosato M, Settanni S, Antocicco M, Battaglia M, Corsonello A, Ruggiero C, et al. Pattern of medication use among older inpatients in seven hospitals in Italy: RESULTS from the CRiteria to assess appropriate medication use among elderly COMPLEX patients (CRIME) project. Curr Drug Saf. (2013) 8:98-103. doi: 10.2174/1574886311308020002

Conflict of Interest: The authors declare that the research was conducted in the absence of any commercial or financial relationships that could be construed as a potential conflict of interest.

Publisher's Note: All claims expressed in this article are solely those of the authors and do not necessarily represent those of their affiliated organizations, or those of the publisher, the editors and the reviewers. Any product that may be evaluated in this article, or claim that may be made by its manufacturer, is not guaranteed or endorsed by the publisher.

Copyright (c) 2021 Lattanzio, Corigliano, Soraci, Fumagalli, Onder, Volpato, Cherubini, Ruggiero, Cozza, Guarasci and Corsonello. This is an open-access article distributed under the terms of the Creative Commons Attribution License (CC BY). The use, distribution or reproduction in other forums is permitted, provided the original author(s) and the copyright owner(s) are credited and that the original publication in this journal is cited, in accordance with accepted academic practice. No use, distribution or reproduction is permitted which does not comply with these terms. 